\title{
Tristetraprolin induces cell cycle arrest in breast tumor cells through targeting AP-1/C-Jun and NF-KB pathway
}

\author{
Li Xu1 ${ }^{1,2, *}$, Huan Ning ${ }^{2, *}$, Ling Gu ${ }^{2}$, Qinghong Wang ${ }^{2}$, Wenbao Lu ${ }^{2}$, Hui Peng ${ }^{2}$, \\ Weiguang Cui ${ }^{2}$, Baoling Ying ${ }^{3}$, Christina R. Ross ${ }^{4}$, Gerald M. Wilson ${ }^{4}$, Lin Wei ${ }^{5}$, \\ William S.M. Wold ${ }^{3}$ and Jianguo Liu ${ }^{2}$ \\ ${ }^{1}$ Department of Respiratory Medicine, The First Affiliated Hospital of Chongqing Medical University, Chongqing, China \\ ${ }^{2}$ Division of Infectious Diseases, Allergy and Immunology, Department of Internal Medicine, Saint Louis University School \\ of Medicine, St. Louis, MO, USA \\ ${ }^{3}$ Department of Molecular Microbiology and Immunology, Saint Louis University School of Medicine, St. Louis, MO, USA \\ ${ }^{4}$ Department of Biochemistry and Molecular Biology, University of Maryland School of Medicine, Baltimore, MD, USA \\ ${ }^{5}$ Department of Immunology, School of Basic Medicine, Hebei Medical University, Shijiazhuang, Hebei, China \\ * These authors have contributed equally to the work
}

Correspondence to: Jianguo Liu, email: jliv9@slu.edu

Keywords: breast cancer, tristetraprolin, cell cycle, AP-1, apoptosis

Received: July 08, $2015 \quad$ Accepted: September 30, $2015 \quad$ Published: October 19, 2015

This is an open-access article distributed under the terms of the Creative Commons Attribution License, which permits unrestricted use, distribution, and reproduction in any medium, provided the original author and source are credited.

\section{ABSTRACT}

The main characteristic of cancers, including breast cancer, is the ability of cancer cells to proliferate uncontrollably. However, the underlying mechanisms of cancer cell proliferation, especially those regulated by the RNA binding protein tristetraprolin (TTP), are not completely understood. In this study, we found that TTP inhibits cell proliferation in vitro and suppresses tumor growth in vivo through inducing cell cycle arrest at the $S$ phase. Our studies demonstrate that TTP inhibits c-Jun expression through the C-terminal $\mathrm{Zn}$ finger and therefore increases Wee1 expression, a regulatory molecule which controls cell cycle transition from the $S$ to the G2 phase. In contrast to the well-known function of TTP in regulating mRNA stability, TTP inhibits c-Jun expression at the level of transcription by selectively blocking NFKB p65 nuclear translocation. Reconstitution of NF-KB p65 completely abolishes the inhibition of c-Jun transcription by TTP. Moreover, reconstitution of c-Jun in TTPexpressing breast tumor cells diminishes Wee1 overexpression and promotes cell proliferation. Our results indicate that TTP suppresses c-Jun expression that results in Wee1 induction which causes cell cycle arrest at the $S$ phase and inhibition of cell proliferation. Our study provides a new pathway for TTP function as a tumor suppressor which could be targeted in tumor treatment.

\section{INTRODUCTION}

Breast cancer is the most common cancer in women and the second leading cause of cancer death (after lung cancer) among women in the United States. The main characteristic of breast cancer is the ability of cancer cells to proliferate uncontrollably $[1,2]$. Though recent progress has broadened our understanding of the mechanisms of tumor progression, the underlying mechanisms of tumor proliferation, especially those regulated by the RNA binding protein tristetraprolin (TTP), are not completely understood.

TTP is one of the best-characterized adenylateuridylate-rich elements (AREs)-binding proteins that mediate mRNA decay, a critical regulation machinery to control the expression of many inflammation- and cancerassociated genes at the level of post-transcription $[3,4]$. TTP promotes rapid mRNA degradation through the ARE motifs present in the 3' untranslated region (3'UTR) of the targeted mRNAs. The mRNAs encoding TNF- $\alpha$, IL- 
23 and GM-CSF are stabilized in TTP-deficient mice and in cells derived from these deficient mice $[3,5,6]$. Overproduction of these cytokines in TTP deficient mice results in a severe systemic inflammatory response including arthritis, autoimmunity and myeloid hyperplasia [7-9]. Collectively, all evidence indicates that TTP is a critical RNA-binding protein in controlling inflammation and maintaining homeostasis. Altered TTP expression may influence the onset and severity of inflammatory syndromes in humans, such as rheumatoid arthritis, systemic lupus erythematosus and ulcerative colitis.

In addition to its impact on inflammation, accumulating evidence in recent years indicates that TTP may behave as a tumor suppressor in diverse neoplastic contexts $[10,11]$. First, TTP expression is suppressed in many human cancers and in cultured cancer cell lines compared to non-transformed tissues or normal cells. Second, TTP expression negatively correlates with breast and prostate cancer progression, and finally, breast cancer patients with low tumor TTP expression show significantly poorer disease-free survival than patients whose tumors express high levels of TTP. The functions of TTP as a tumor suppressor are mediated through rapid decay of mRNAs encoding molecules associated with tumorigenesis, including VEGF [12], COX2 [13], HIF-1 [14], MMP1 and uPA/uPAR [15]. Currently, TTP is best known for its function to promote mRNA decay through the ARE in the 3'UTR. It remains elusive whether TTP controls tumor-promoting gene transcription in tumor cells.

Transcription factors, such as AP-1 and NF- $\kappa$ B, play a variety of roles in tumor cell survival, differentiation and proliferation [16]. Dysregulation of NF- $\mathrm{B}$ activity has been linked to cancer, inflammatory and autoimmune diseases, viral infection, and improper immune development $[17,18]$. The transcription factor c-Jun is a proto-oncogene and a critical member of the activator protein-1 (AP-1) complex. The AP-1 complex is composed of homodimers of Jun family members (c-Jun, JunB and JunD), heterodimers of Jun and Fos (c-Fos, FosL1, FosL2, and FosB), or cAMP response element-binding protein (CREB)/activating transcription factor (ATF) family members. In breast cancer cells, AP-1 proteins have been identified as critical regulators for transformation, growth and invasion [19, 20]. Expression of c-Jun has been reported to have prognostic value in breast cancers and several other tumor types [21, 22]. Overexpression of c-Jun in breast cancer cells is associated with endocrine resistance, and increases migration/invasion in vitro and tumor formation in vivo [23], while the cells expressing dominant-negative c-Jun fail to invade [24, 25]. However, it is largely unknown whether TTP regulates c-Jun expression in breast tumor cells and the role of NF- $\mathrm{KB}$ in TTP-mediated c-Jun expression.

In this study, we found that expressing TTP in breast tumor cells inhibits cell proliferation in vitro and breast tumor growth in vivo. TTP does not directly cause apoptosis, rather it induces cell cycle arrest at the $\mathrm{S}$ phase. Furthermore, our studies demonstrate that TTP inhibits c-Jun expression that leads to an increase in Wee1 expression, a regulatory molecule controlling cell cycle transition from the $\mathrm{S}$ to the $\mathrm{G} 2$ phase. Inhibition of c-Jun by TTP is mediated through blocking NF- $\kappa B$ p65 nuclear translocation rather than affecting mRNA stability. Importantly, reconstitution of c-Jun in TTPexpressing cells abolishes Wee1 induction and promotes cell proliferation. Our results indicate, for the first time, that TTP inhibits c-Jun transcription by blocking NF- $\kappa B$ p65 nuclear translocation that results in Wee1 induction and cell cycle arrest at the $\mathrm{S}$ phase, leading to suppression of breast tumor cell proliferation.

\section{RESULTS}

\section{TTP suppresses breast tumor growth}

TTP expression has been previously reported to be low in a multitude of tumors, with low TTP being associated with poor survival of cancer patients [11]. To determine the direct effects of TTP on cancer cell proliferation, we expressed TTP with TTP-expressing adenovirus in breast tumor cells. TTP was strongly induced after adenoviral infection (Figure 1A). Overexpression of TTP significantly inhibited MCF7 cell growth (Figure 1B). In order to tightly control TTP expression in vivo and in vitro, we generated inducible TTP expression in MDA-MB-231 cells using the Tet-Off system. TTP was modestly expressed in the presence of low concentration of Dox (Dox +$)$ and strongly induced after withdraw of Dox (Dox-) in culture medium (Figure 1C). As a result of TTP induction, the proliferation of MDA-MB-231 cells was inhibited by TTP in a dose-dependent manner (Figure 1D). Next, we induced TTP expression in the TTP/TetOff MDA-MB-231 cells and then inoculated the cells into mammary gland pats of the NSG mice. Consistent with the in vitro data, all NSG mice that received TTP-expressing tumor cells did not develop tumor, while mice that received tumor cells with empty vector (EV) developed rapid-growing tumors (Figure $1 \mathrm{E} \& 1 \mathrm{~F}$ ). Meanwhile, the expression of TTP in tumors of mice that received TTP/ Tet-Off MDA-MB-231 cells was confirmed by Western blot with an anti-FLAG antibody against the Flag-tagged TTP protein (Figure 1G). These results indicate that TTP inhibits tumorigenesis of breast cancer.

\section{TTP inhibits tumor cell proliferation through causing cell cycle arrest at the $S$ phase}

To understand the mechanisms of TTP-mediated inhibition of cell proliferation, we first examined apoptosis 
A.

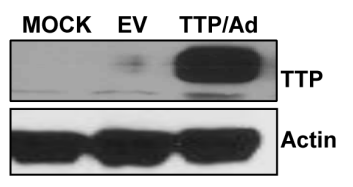

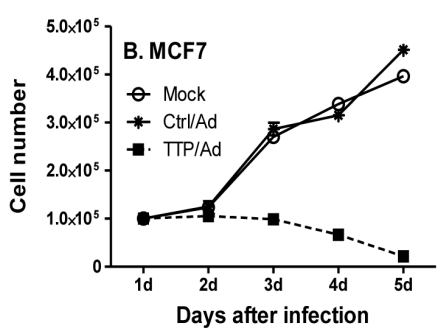

Days after infection
C.

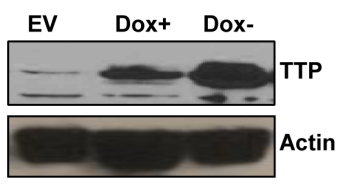

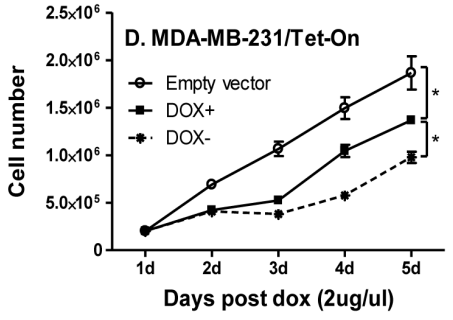

G.

F.

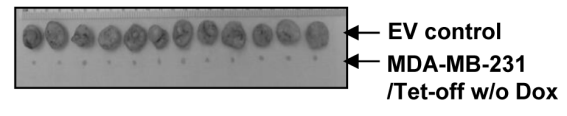

EV1 EV2 EV3 T1 T2 T3 PO

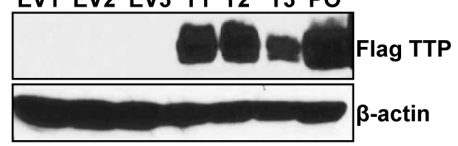

Figure 1: TTP inhibits breast cancer cell proliferation and tumor development. MCF7 cells were infected with TTP/ adenovirus and control adenovirus at MOI $=1$. TTP expression was detected $24 \mathrm{~h}$ after infection by Western blot with anti-TTP antibody A., and cell numbers were counted every $24 \mathrm{~h}$ until 5 days after infection B. Results shown are mean plus SEM of three independent experiments with each run in duplicate. $1 \times 10^{5} \mathrm{TTP} /$ Tet-Off MDA-MB-231 cells were cultured with or without $2 \mu \mathrm{g} / \mathrm{ml}$ doxycycline (Dox). TTP expression was measured by western blot 5 days after withdraw Dox C., and cell counting was performed at indicated times D. TTP/ Tet-Off MDA-MB-231 cells were cultured for one week without Dox, and then $5 \times 10^{6} \mathrm{TTP} /$ Tet-Off MDA-MB-231 were inoculated s.c. into mammary glands of the NSG mice. Tumor growth was measured and recorded $\mathbf{E}$. Tumors were excised at day 29 after tumor cell inoculation and representative tumors for each experimental group were shown F., G. Tumor tissues were lysed and total proteins were extracted for detecting Flag-tagged TTP levels by western blot with anti-FLAG antibody. EV: tumors induced with Tet-off cells expressing empty vector; T: tumors generated with Tet-off cells expressing TTP. Number means the number of tumors.
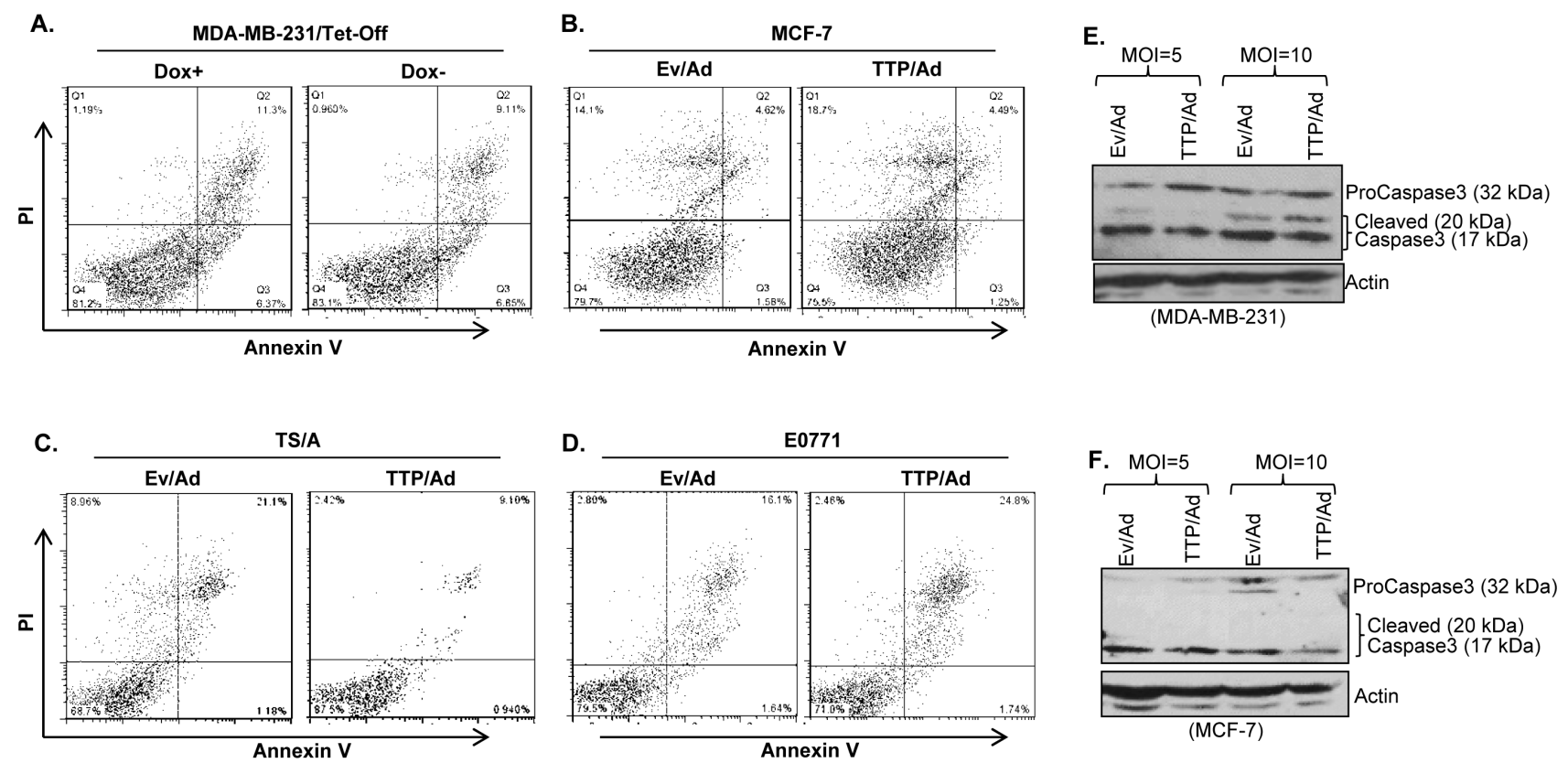

Figure 2: TTP does not induce apoptosis of breast tumor cells. Apoptosis was measured by flow cytometry in MDA-MB-231/ Tet-Off cells 72 hours after withdrawing Dox in culture medium A. MCF7 cell B., TS/A C. and E0771 D. breast tumor cells were infected with control adenovirus (Ev/Ad) or TTP-expressing adenovirus (TTP/Ad) at MOI=10 for 96 hours, followed by measuring apoptosis by FACS. Caspase 3 and its cleaved products were measured by immunoblotting with anti-Caspase 3 antibody in MDA-MB-231 E. and in MCF7 F. cells after TTP/Ad infection with indicated MOI. Actin serves as loading control. 
in cells infected with TTP-expressing adenovirus. As shown in Figure 2A-2D, TTP had no direct effect on apoptosis (indicated as Annexin and PI positive cells) in human and mouse breast cancer cell lines after expressing TTP by adenovirus. In addition, there was no difference in the expression of cleaved Caspase 3 in MDA-MB-231 cells (Figure 2E) or in MCF7 cells (Figure 2F) after expressing TTP by adenovirus. These data are consistent with previous reports [11] that TTP itself does not induce apoptosis rather increases the sensitivity of cells to apoptotic insults.

Next, we wondered whether TTP inhibits cell proliferation through regulating cell cycle. Indeed, TTP expression caused cell cycle arrest at the $\mathrm{S}$ phase in MDAMB-231 cells (Figure 3A) and in MCF7 cells (Figure 3E). Compared to the cells infected with control adenovirus (EV/Ad), the percentages of cells in the $\mathrm{S}$ phase were increased over 30\% after expressing TTP in MDAMB-231 cells (Figure 3B) and promoted from $20 \%$ to $80 \%$ in MCF7 cells (Figure 3F). These data indicate that TTP suppresses breast tumor cell proliferation through inducing cell cycle arrest. To understand the mechanisms of TTPinduced cell cycle arrest, we detected the expression of Wee1, one of the key regulators in control of cell cycle transition from the $\mathrm{S}$ into $\mathrm{G} 2 / \mathrm{M}$ phase. We found that Wee1 mRNA and protein expression was up-regulated in TTP-expressing MDA-MB-231 cells (Figure 3C \& 3D) and in MCF7 cells (Figure 3G \& 3H). Since Wee1 blocks cell cycle transition from the $\mathrm{S}$ into $\mathrm{G} 2 / \mathrm{M}$ phase, an increase in Wee1 expression can result in cell cycle arrest at the $\mathrm{S}$ phase.

\section{TTP inhibits c-Jun expression in breast cancer cells}

The cell cycle is tightly regulated by many molecules, including transcription factor c-Jun [31, 32]. To determine whether TTP affects c-Jun expression, we first expressed TTP and then measured c-Jun in several breast cancer cell lines. TTP expression inhibited c-Jun mRNA expression in MDA-MB-231 (Figure 4A), T47D (Figure 4B) and MCF7 (Figure 4C) cells. In agreement with the suppressive effects of TTP on c-Jun expression, deletion of TTP increased c-Jun protein expression in mouse embryonic fibroblasts (Figure 4D). We and others have previously shown that TTP controls target gene expression through affecting their mRNA stability. So we measured the half-life of c-Jun mRNA in cells expressing TTP. Intriguingly, the half-life of c-Jun mRNA was not affected by TTP expression in MDA-MB-231 cells (Figure 4E) and in MCF7 (Figure 4F) cells, indicating that TTP suppression of c-Jun expression is not mediated at the post-transcriptional level.
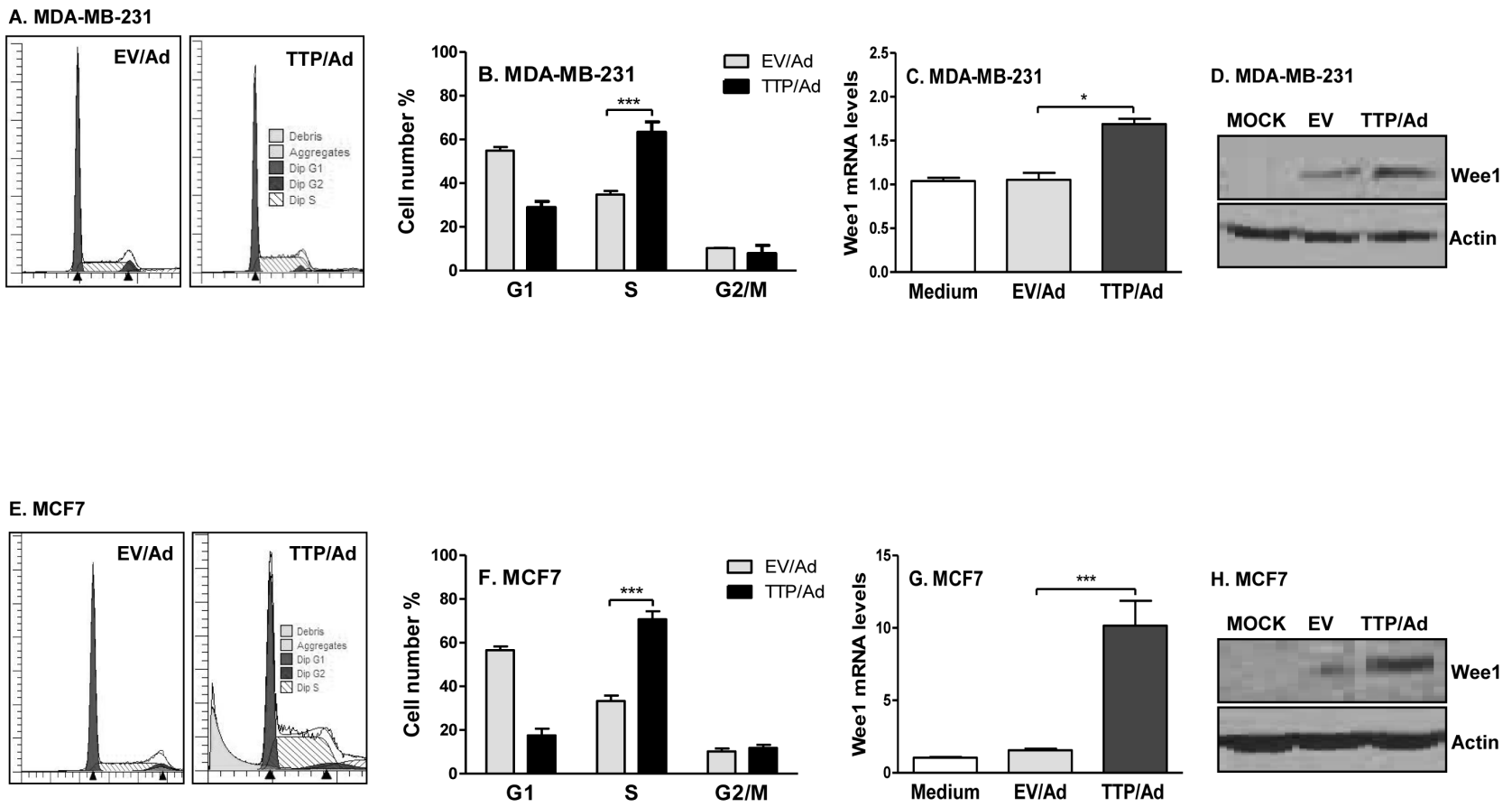

Figure 3: TTP causes cell cycle arrest at the $\mathbf{S}$ phase and induces Wee1 expression. $5 \times 10^{6} \mathrm{MDA}-\mathrm{MB}-231 \mathrm{~A} .-\mathrm{D}$. and MCF7 cells E.-H. cells were infected with TTP/adenovirus (TTP/Ad) and control adenovirus (EV/Ad). After 48 h, cells were harvested and PI added for FACS analysis. Data shown are one representative of three experiments in MDA-MB-231 A. and MCF7 E. cells of the FACS data, and mean plus SD of three experiments in MDA-MB-231 B. and MCF7 F. cells. Wee1 mRNA C. \& G. and protein D. \& H. levels were measured in MDA-MB-231 and MCF7 cells in above conditions. 


\section{TTP inhibits c-Jun transcription via its zinc finger domain}

Since TTP-mediated c-Jun inhibition is not at the level of post-transcription, we wanted to know whether TTP inhibits c-Jun expression at the transcriptional level. We measured the nascent primary transcript rate of c-Jun by qRT-PCR using a pair of primers corresponding to the intron 1 and the exon 2 region of the human c-Jun gene after expressing TTP. TTP strongly inhibited c-Jun primary gene transcript in MDA-MB-231 (Figure 5A) and MCF7 (Figure 5C) cells, which was inversely correlated with TTP expression (Figure 5B \& 5D). Furthermore, suppressing TTP expression in TTP/Tet-Off MDAMB-231 cells by adding Dox increased c-Jun primary transcript in a time-dependent manner (Figure 5E \& 5F). These data indicate a transcriptional inhibition of c-Jun by TTP. Moreover, we generated a human c-Jun promoterdriving luciferase reporter construct and co-transfected this vector with different amounts of TTP expression vector into HEK293 cells, followed by measuring luciferase activity. TTP dose-dependently inhibited c-Jun promoter-driving luciferase activity (Figure $5 \mathrm{G}$ ), further confirming that TTP inhibits c-Jun mRNA expression at the level of transcription. Structurally, TTP is a zinc-finger protein having two tandem zinc-finger domains. The zinc finger has been found in many RNA-binding proteins responsible for binding to the 3'UTR of different mRNAs [33-35]. To determine which domain is responsible for TTP inhibition of c-Jun, we co-transfected two TTP zinc finger mutants, TTP/C124R and TTP/C147R, with c-Jun promoter-driving luciferase reporter plasmid into HEK293 cells, followed by measuring luciferase activity. As shown in Figure 5H, the C147R mutant lost its suppressive effect on c-Jun promoter activity, while the C124R mutant still did. This C147R dependent inhibition was also shown at the protein level, in that TTP-mediated c-Jun protein inhibition was abolished in cells transfected with the C147R mutant but the C124R mutant (Figure 5I). These data demonstrate that TTP via the C-terminal $\mathrm{Zn}$ finger inhibits c-Jun transcription.

\section{TTP inhibits c-Jun transcription through affecting NF-kB pathway}

Ours and others study showed that, in addition to the RNA destabilization effect, TTP can inhibit NF-кB nuclear translocation and consequently affect
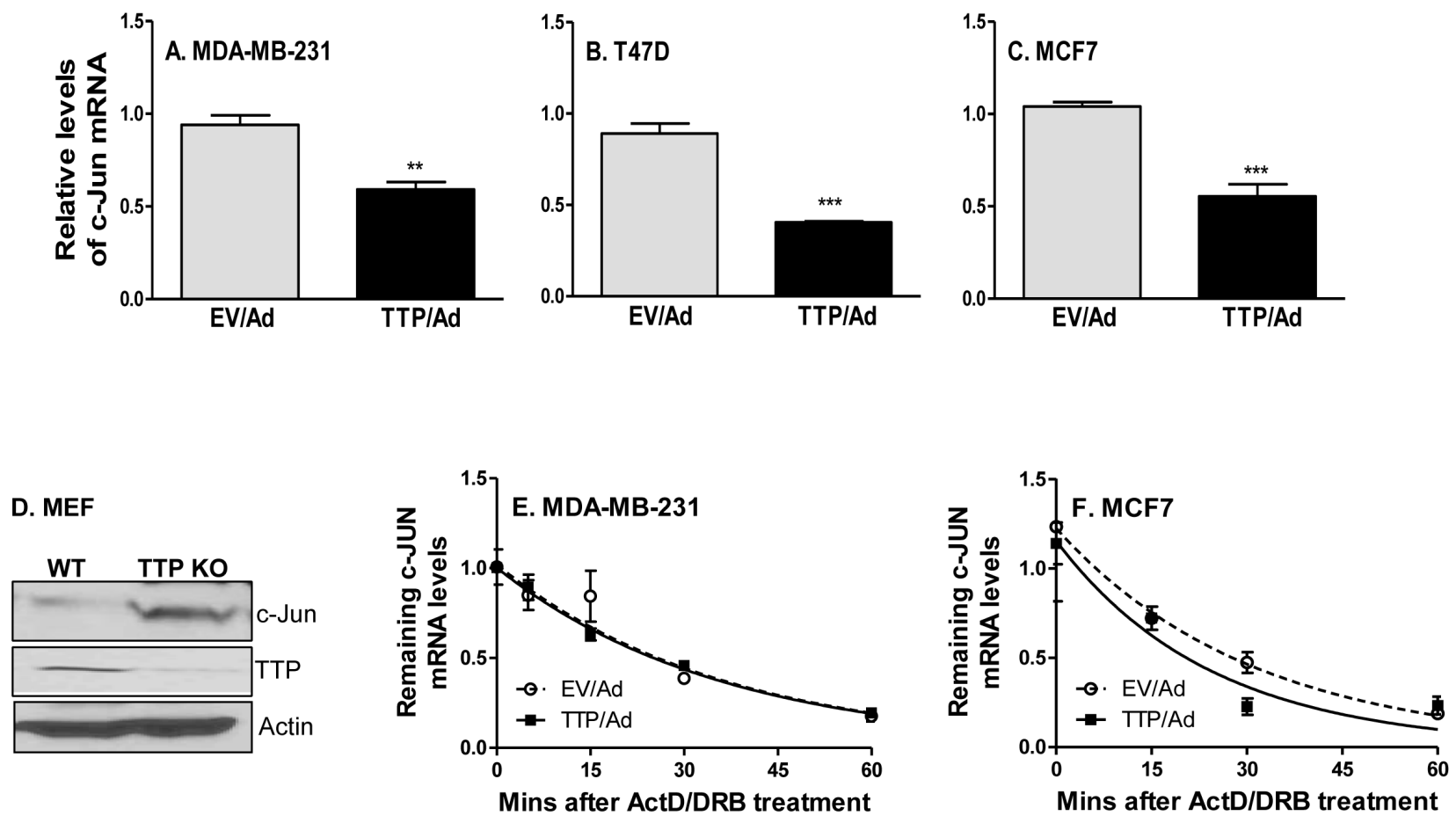

Figure 4: TTP inhibits c-Jun expression at the level of transcription. MDA-MB-231 A., T47D B. and MCF7 C. tumor cells were infected with TTP/adenovirus (TTP/Ad) and control adenovirus (EV/Ad). After 48 hours, total RNA was extracted to detect c-Jun expression by real time RT-PCR. c-Jun protein was detected by western blot with whole cell lysates extracted from TTP $/$ (KO) and WT MEF cells D. The half-life of c-Jun mRNA was analyzed in MDA-MB-231 E. and MCF7 cells F. at 24 hours after TTP/Ad and control adenovirus (EV/Ad) infection. Total RNA was isolated as indicated time points after adding Actinomycin D $(10 \mu \mathrm{g} / \mathrm{ml})$, and remaining c-Jun mRNA were measured using real-time RT-PCR. The level of c-Jun mRNA before added ActD was set at 1.0. Each point is represented as mean plus SEM. 
downstream cytokine expression [36-38]. To determine whether TTP affects NF-кB expression in breast tumor cells, we measured nuclear NF-кB in cells infected with TTP/adenovirus in the presence or absence of TNF- $\alpha$, a strong inducer of NF-кB. As shown in Figure 6A, TTP expression inhibited nuclear p65 and c-Rel but not p50 (left panel), while had no effects on cytoplasmic NF-кB expression (right panel) in MDA-MB-231 cells. TNF- $\alpha$ treatment induced nuclear expression of p65 and c-Rel in cells infected with control virus but not in cells expressed TTP (left panel, Figure 6A). To confirm the effects of TTP on nuclear NF-кB expression, we examined NF-кB levels in WT and $\mathrm{TTP}^{-/-} \mathrm{MEF}$ cells with or without TNF- $\alpha$ treatment. The expression of nuclear p65 and c-Rel was increased in $\mathrm{TTP}^{-/-} \mathrm{MEF}$ cells compared with WT MEF cells, especially at 1 hour after TNF- $\alpha$ treatment, while the cytoplasmic NF-кB levels did not change significantly (Figure 6B). These results indicate that TTP inhibits nuclear expression of p65 and c-Rel in breast tumor cells.

Next, we co-transfected c-Jun promoter-luciferase vector and TTP expression vector with three members of the NF-кB family into HEK293 cells, followed by measurement of luciferase activity. Overexpression of TTP inhibited c-Jun promoter activation. Interestingly, this inhibition was almost completely abolished when the p65 but not p50 and c-Rel was introduced into the cells (Figure $6 \mathrm{C}$ ), suggesting that the inhibitory effects of TTP on c-Jun expression is mediated through NF- $\mathrm{KB}$ p65. To confirm successful expression of the TTP and NF-кB family members after transfection, we measured protein levels of TTP and NF-кB p65, p50, and c-Rel by Western blot from the transfected cells. As shown in Figure 6C (lower panel), protein expression of ТTP, NF-кB p65, c-Rel, and p50 was markedly increased compared with their respective controls. To confirm further the role of NF-KB in c-Jun transcription, we co-transfected c-Jun promoter-luciferase vector with an I $\mathrm{I} B-\alpha$ dominant positive mutant which prevents disassociation of $\mathrm{NF}-\kappa \mathrm{B}$ from the I $\mathrm{B}$ complex and therefore inhibits $\mathrm{NF}-\kappa \mathrm{B}$ nuclear translocation, followed by measurement of luciferase activity. Inhibition of NF- $\kappa \mathrm{B}$ by the I $\kappa \mathrm{B}-\alpha$ mutant dramatically suppressed c-Jun promoter activity (Figure 6D). Taken together, these results suggest that TTP suppresses c-Jun transcription through inhibition of nuclear p65 expression.
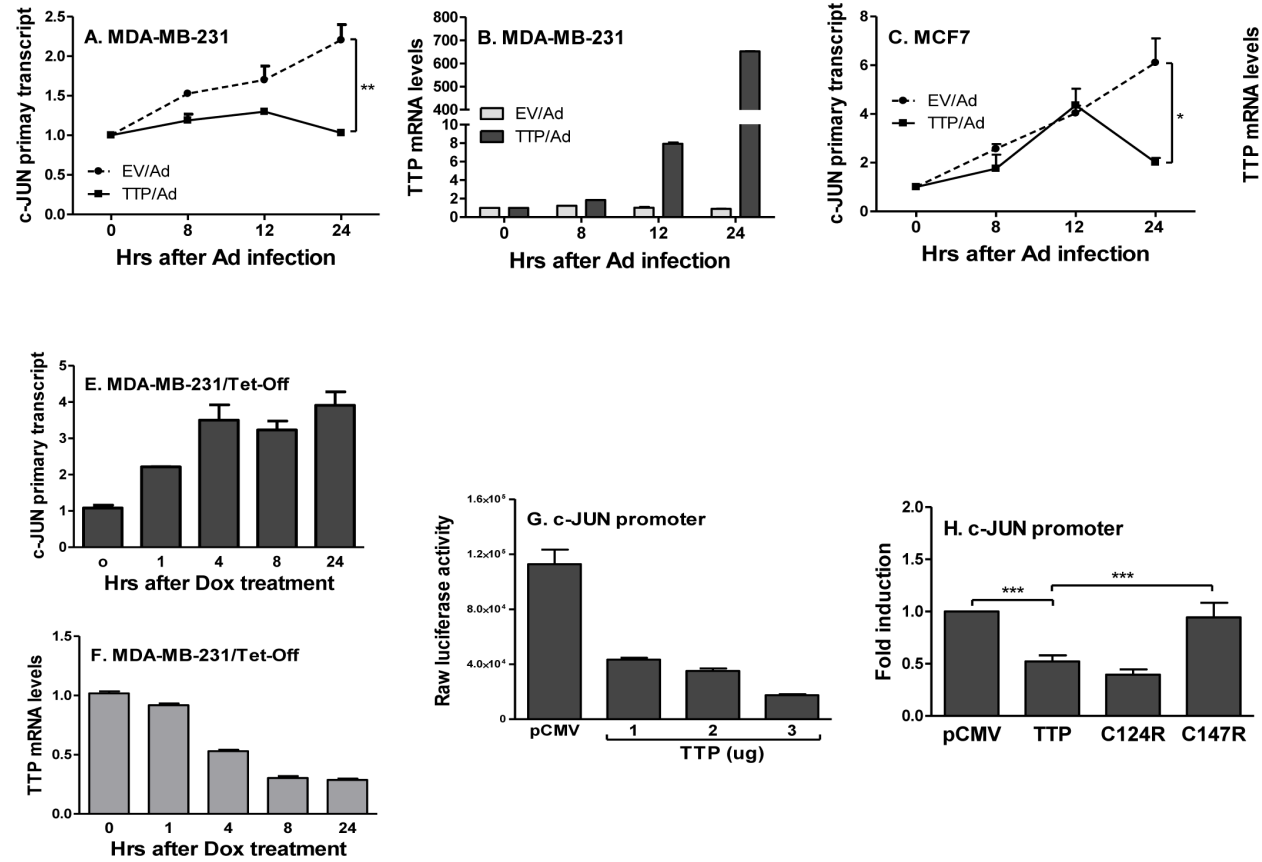

I.

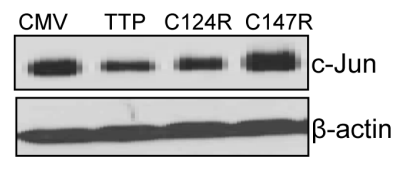

Figure 5: TTP inhibits c-Jun transcription via zinc finger at $\mathbf{1 4 7 .}$ MDA-MB-231 A. and MCF7 C. cells were infected with TTP/Ad and control adenovirus (EV/Ad) for different times as indicated, followed by extraction of total RNA to measure c-Jun primary transcript by real-time RT-PCR. The TTP expression in the above MDA-MB-231 B. and MCF7 D. cells were determined by real-time RTPCR. c-Jun E. and TTP mRNA F. was measured by real time PCR in the TTP/Tet-Off MDA-MB-231 cells after adding Dox ( $2 \mu \mathrm{g} / \mathrm{ml})$ for different times as indicated. Data represent are mean plus SEM of three experiments. $5 \times 10^{6}$ HEK293 cells were transiently transfected with c-Jun promoter-driven luciferase construct along with different amounts of TTP expression vector, followed by measurement of luciferase activity in cell lysates G. $5 \times 10^{6}$ HEK293 cells were transiently transfected with c-Jun promoter-driven luciferase construct along with TTP as well as two TTP zinc finger mutant constructs (C124R \& C147R), followed by measurement of luciferase activity in cell lysates after 24 hours H. Data shown as relative levels compared to luciferase activity in cells transfected with the empty vector (pCMV). c-Jun protein levels were detected in the above cells by western blot with antibody against c-Jun protein $\mathbf{I}$. 


\section{c-Jun/Wee1 axis is involved in TTP-mediated cell cycle arrest}

Since TTP inhibits c-Jun and increases Weel expression in breast tumor cells, we tested whether TTP increases Weel expression through inhibiting c-Jun. We overexpressed c-Jun in breast tumor cells and then measured Wee1 expression. Overexpression of c-Jun significantly inhibited Wee1 expression in MDA-MB-231 (Figure 7A) and MCF7 (Figure 7C) cells. Importantly, the increased Wee1 expression was reduced to baseline after introducing c-Jun into TTP-expressing MDA-MB-231 (Figure 7B) and MCF7 (Figure 7D) cells, indicating a regulatory axis of TTP/c-Jun/Weel. In addition, cell proliferation was also recovered after introducing c-Jun into the cells expressing TTP (Figure 7E). These results indicate that TTP-induced cell cycle arrest is mediated by
c-Jun/Wee1 axis.

\section{DISCUSSION}

TTP is an RNA-binding protein important for maintenance of homeostasis through regulating expression of proinflammatory cytokines. Recent emerging evidence indicates that TTP also plays a crucial role in tumor progression. TTP mRNA and protein levels were found to be significantly decreased in breast tumors compared with non-transformed tissue samples, and low TTP is associated with poor prognosis in breast cancer patients [39]. A few studies show lower or undetectable levels of TTP in breast cancer cell lines or in highly invasive cell lines than in normal mammary gland epithelial cell lines [39-41]. So far, most studies were performed with in vitro system. Using inducible expression of TTP with the Tet-Off system, our in vivo experiments show that TTP expression

\section{A. MDA-MB-231}

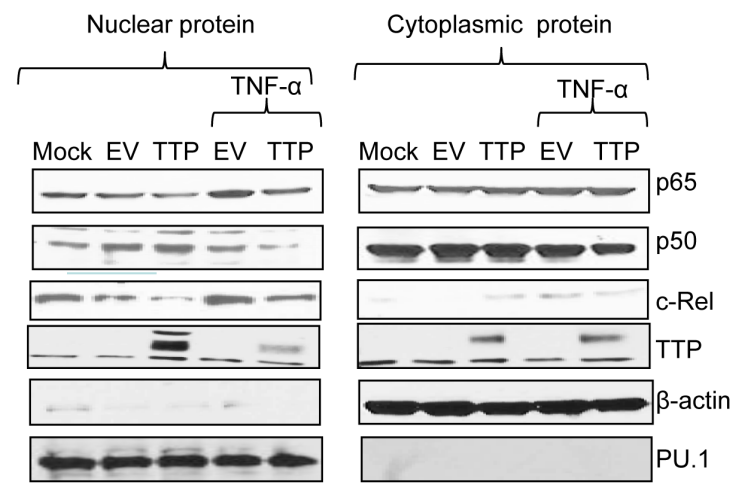

\section{B. MEF}

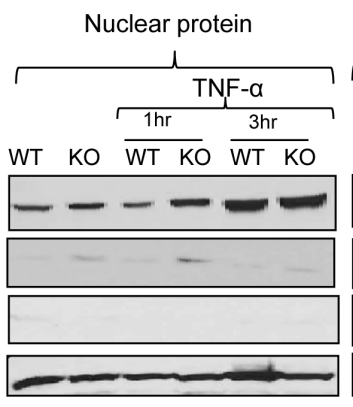

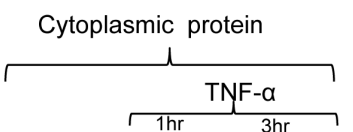

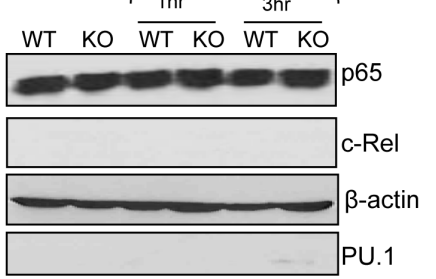

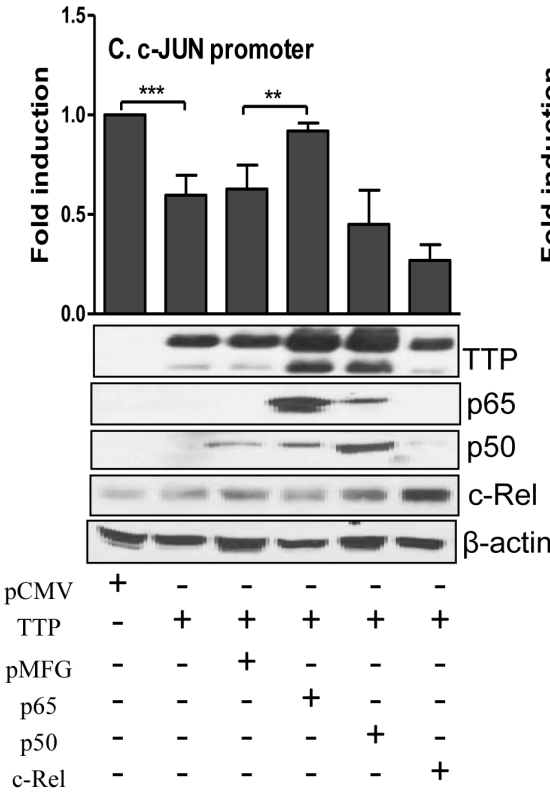

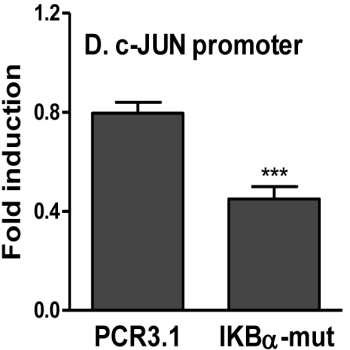

Figure 6: TTP inhibits NF-KB nuclear translocation. $5 \times 10^{6}$ MDA-MB-231cells were infected with TTP/Ad for 24 hours and then stimulated with or without $5 \mathrm{ng} / \mathrm{ml}$ of TNF- $\alpha$ for $30 \mathrm{~min}$, followed by nuclear and cytoplasmic protein extraction to measure TTP, p65, p50 and c-Rel by Western Blot with their respective antibodies A. Image represents one of three experiments with similar results. $5 \times 10^{6} \mathrm{TTP}^{-/-}$ and WT MEF cells were treated with or without $30 \mathrm{ng} / \mathrm{ml} \mathrm{TNF}-\alpha$ for $1 \mathrm{~h}$ and $3 \mathrm{~h}$, followed by nuclear and cytoplasmic protein extraction to measure TTP, p65 and c-Rel by Western Blot. $\beta$-actin and PU.1 were used as control for cytoplasmic and nuclear protein B. $5 \times 10^{6} \mathrm{HEK} 293$ cells were transiently transfected with c-Jun promoter luciferase construct along with TTP and NF- $\mathrm{kB}$ expression vectors, followed by measurement of luciferase activity by luminometer $\mathbf{C}$. The data shown were normalized to the results obtained from CMV empty cotransfection group. Results shown are mean plus SD of four independent experiments. The transfected cells of each condition were lysed for

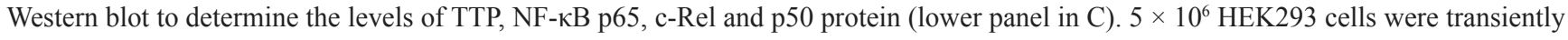
transfected with c-Jun promoter construct along with IkB- $\alpha$ mutant and its empty vector PCR3.1, followed by measurement of luciferase activity by luminometer $\mathbf{D}$. Results shown are mean plus SD of three independent experiments. 
completely inhibits breast tumor development (Figure $1 \mathrm{E} \& 1 \mathrm{~F})$, confirming that TTP is a tumor suppressor. Though TTP suppresses breast cancer cell proliferation, we observed variable effects of TTP on $\mathrm{ER}^{+}$and ER- tumor cells. In $\mathrm{ER}^{+} \mathrm{MCF} 7$ cells, we even observed cell death after TTP expression by adenovirus (data not shown). In ER- MDA-MB-231 cells, we didn't observe cell death after TTP being induced even for 6 days (data not shown). Barrios-Garcia et al recently reported that MCF7 cells overexpressing TTP exhibit a significant reduction in ER $\alpha$ dependent transcriptional activation and in E2-induced cell proliferation [42]. This could be the reason why TTP induces stronger inhibition of cell proliferation, even cell death, in ER positive cells (MCF7) than in ER negative cells (MDA-MB-231). When TTP was expressed to much higher levels in both $\mathrm{ER}^{+}$and $\mathrm{ER}^{-}$breast tumor cells by lentivirus, we observed cell death in both cell lines (data not shown), indicating that high levels of TTP induce cell death independent of the ER $\alpha$ status in breast cancer cells.

We found that TTP does not directly induce apoptosis of breast tumor cells, which is consistent with previous report that TTP sensitizes tumor cells to some apoptotic signals [11]. Intriguingly, after expressing TTP with adenovirus for 48 and 72 hours, we observed almost all cells were arrested at the $\mathrm{S}$ phase (Figure 3A-3E). We expressed TTP in normal mammary gland epithelial cells and observed that TTP did not inhibit growth of normal mammary gland epithelial cells (data not shown), indicating that TTP specifically induces cell cycle arrest of breast tumor cells. In addition, different amounts of TTP seem targeting different pathways for cell growth suppression. It has been reported that TTP can target G1 to $\mathrm{S}$ phase cell cycle control genes, such as p21 [43], cyclin D1 [44], E2F1 [45] and LATS2 [46]. The effects of TTP on these G1/S cell-cycle genes are probably mediated through its destabilizing effects on mRNA. In this study, we found, for the first time, that TTP affects the S/G2 cell cycle transition but not the G1/S cell cycle checkpoint in breast tumor cells. Progression from the $\mathrm{S}$ to the G2/M phase is a complex process, and involves cde 2 and cyclinB which are regulated positively by cdc 25 phosphatase and negatively by Wee1 kinase. Wee1 was first identified in fission yeast, where Wee1 deficiency caused premature mitotic entry and replication of smaller-sized yeast [47]. Wee1 inhibits phosphorylation of the tyrosine 15 residue on $\mathrm{Cdk} 1 / \mathrm{Cdc} 2$, close to its ATP-binding pocket [48]. Wee1 also modulates the activity of Cdk $1 / 2$ through inhibitory phosphorylation of conserved tyrosine 15 residues on both kinases, thereby controlling entry into mitosis and DNA replication during $\mathrm{S}$ phase. Therefore, early preclinical and clinical studies have focused on the modulation of Wee1 activity and abrogation of the G2 checkpoint in the presence of DNA damaging agents, utilizing the concept of mitotic lethality as a mechanism of antitumor activity. In
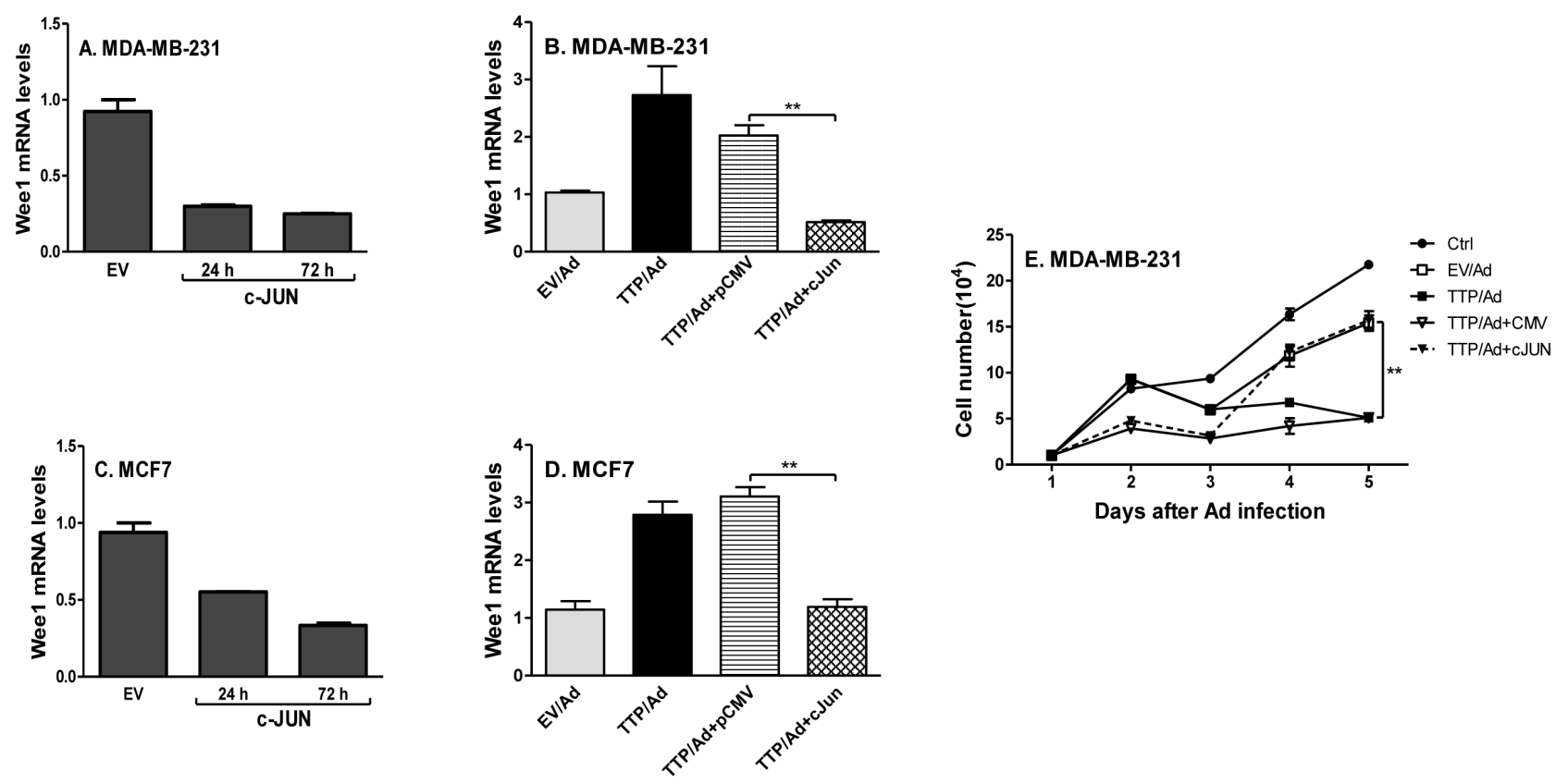

Days after Ad infection

Figure 7: TTP inhibits cell proliferation through down-regulating c-Jun and up-regulating Wee1 expression. MDAMB-231 A. and MCF7 C. cells were transfected with c-Jun expression vector and empty vector for $24 \mathrm{~h}$ and $72 \mathrm{~h}$, followed by measuring Wee1 mRNA with real time PCR. MDA-MB-231 B. and MCF7 D. cells were infected with TTP/Ad and control adenovirus (EV/Ad) for 48 $\mathrm{h}$ and then transfected with c-Jun expression vector or empty control vector for additional $24 \mathrm{~h}$, followed by measuring Wee $1 \mathrm{mRNA}$ by real time PCR. For each experiment, real-time PCR assays were performed three times and each point represented by mean plus SEM. MDAMB-231 cells were infected with TTP/adenovirus for $12 \mathrm{~h}$ and then transfected with c-Jun expression vector or empty vector, followed by counting cell numbers at different times as indicated $\mathbf{E}$. Data shown are mean plus SD from triplicate cells of three experiments. 
this study, we observe that TTP induces Weel expression in breast cancer cells which can prevent cell progression into the G2 stage and cause cell cycle arrest at the $\mathrm{S}$ phase. c-Jun is overexpressed in most tumors and defined as a proto-oncogene. In this study, we have provided evidence indicating that TTP inhibits c-Jun expression at the transcriptional level. Our study indicates that the impaired TTP expression in tumor cells may be accountable for c-Jun overexpression. In addition to its mRNA decay role, TTP has been shown to act as a transcriptional co-repressor in regulating NF- $\kappa \mathrm{B}-$ dependent transcription [38, 41, 49]. TTP can affect $\mathrm{NF}-\kappa \mathrm{B}$ translocation [38] and physically interacts with the p65 subunit of NF- $\mathrm{B}$ [41, 49]. In this study, we demonstrate that in breast tumor cells TTP inhibits c-Jun expression through affecting nuclear expression of the $\mathrm{NF}-\kappa \mathrm{B}$, especially the p65 subunit. Collectively, we have revealed a function for TTP as a tumor suppressor through down-regulation of pro-oncogene c-Jun expression. To our knowledge, this is the first report showing a correlation between TTP and c-Jun/AP-1 expression and the underlying mechanisms. The inverse correlation between c-Jun and Wee1 in TTP-expressing cells led us to hypothesize that c-Jun may regulate Weel expression. Indeed, c-Jun significantly reduces Weel expression, indicating a direct correlation between c-Jun and Weel in breast tumor cells. On the other hand, the increased Wee1 expression in TTP-expressed cells was reduced to basal level when c-Jun was expressed (Figure 7A-7D), plus cell growth was also recovered after c-Jun was introduced in the above cells (Figure 7E), further establishing the axis of TTP/c-Jun/Wee1 in regulation of cell proliferation. Shruti Lal et al provided evidence that posttranscriptional regulation of WEE1 by $\mathrm{Hu}$ antigen (HuR) is critical for human pancreatic ductal adenocarcinoma cell survival under clinically relevant drug exposure [50]. HuR and TTP are both RNA binding proteins with opposite effects. HuR stabilizes and TTP destabilizes mRNA of target genes, respectively. It will be interested in investigating the role of HuR in TTP-mediated induction of Weel expression in breast cancer cells in further exploration.

In summary, our study demonstrates that TTP, as a transcriptional co-suppressor, inhibits c-Jun expression independent of its mRNA destabilization function, consequently resulting in increase in Weel expression and preventing cell cycle progression from the $\mathrm{S}$ phase into the G2 phase. TTP suppresses c-Jun transcription through selectively blocking nuclear translocation of NF$\kappa \mathrm{B}$ p65. Our study reveals that the c-Jun/Wee1 axis is an important target for TTP in breast cancer development, and the findings have therapeutic potential for developing treatment of tumor patients.

\section{MATERIALS AND METHODS}

\section{Mice}

6 8 week old NSG mice were obtained from The Jackson Laboratories and housed in cages with filter tops in a laminar flow hood, fed food and acid water ad libitum in pathogen-free condition. All experimental procedures were performed with approval of the IACUC at Saint Louis University. For tumor induction, $5 \times 10^{6} \mathrm{MDA}-$ MB-231/Tet-Off and empty vector control cells in $100 \mu \mathrm{l}$ phosphate-buffered saline were injected subcutaneously (s.c.) into the abdominal mammary glands of NSG mice. Tumor volume was calculated by the formula length $\times$ width $\times$ height $\left(\mathrm{mm}^{3}\right)$.

\section{Cells}

All human breast cancer cell lines, MCF7, MDAMB-231, MDA-MB-453 and T47D cells, as well as HEK 293 cells and HEK 293 T cells were obtained from the American Type Culture Collection (Manassas, VA). Cells were maintained in Dulbecco's modified Eagle's medium or RPMI 1640 supplemented with 2 mM glutamine, 100 $\mathrm{U} / \mathrm{ml}$ penicillin and streptomycin, and $10 \%$ FBS (SigmaAldrich, St. Louis, MO; endotoxin, N-myristoyltransferase at 10.0 endotoxin units $/ \mathrm{ml}$ ). Wild-type (WT) and TTP knockout (KO) mouse embryonic fibroblasts (MEF) were kindly provided by Perry J. Blackshear.

\section{Plasmids and reagents}

The expression vectors for TTP, CMV hTTP. Flag, CMV hTTP/C124R, CMV hTTP/C147R mutant, were kindly provided by Perry J. Blackshear [26]. The expression vectors for NF-кB p50, p65, and c-Rel were originally provided by K. Murphy (Washington University, St. Louis, MO) [27]. The human c-Jun promoter construct was PCR synthesized using primers: forward 5'GAGGTTTGATTTACGCAT 3' and reverse 5' ATGTGCTGTGACCATTTA 3', from -444 to +191 sites, with MDA-MB-231 cell genomic DNA as template. The PCR product was cloned into pGL2 Basic (Promega) reporter vector between HindIII and KpnI sites. The IкBmutant plasmid was described previously [28]. All plasmid DNAs were prepared with Qiagen EndoFree Maxiprep kits. Rabbit anti-TTP Ab (N-terminal) was purchased from Sigma-Aldrich. Rabbit anti-NF-кB p65, c-Rel, p50 and c-Jun mAbs were purchased from Santa Cruz Biotechnology (Santa Cruz, CA). Rabbit anti-Flag mAb was obtained from Roche Applied Science (Indianapolis, IN). Recombinant human TNF- $\alpha$ was purchased from PeproTech (Rocky Hill, NJ). Unless stated otherwise, all 
other chemicals were obtained from Sigma-Aldrich.

\section{Cell Extracts and western blotting}

Cells were grown and transfected with NF-кB p65, c-Rel, and p50 expression plasmids. Thirty-six hours after transfection, cells were stimulated with TNF- $\alpha$ (5 $\mathrm{ng} / \mathrm{ml}$ for MDA-MB-231 and $30 \mathrm{ng} / \mathrm{ml}$ for MEF cells) for different times $(0.5,1 \& 3 \mathrm{~h})$. Then, cytoplasmic and nuclear extracts were harvested as described previously [29]. Proteins $(100 \mu \mathrm{g})$ were separated by $10 \%$ SDS-PAGE, transferred to polyvinylidene difluoride membranes, and blocked in 5\% nonfat milk in Tris buffer ( $\mathrm{pH}$ 8.0). Primary Ab was added at a concentration of 1 $\mu \mathrm{g} / \mathrm{ml}$ in blocking buffer and left overnight at $4^{\circ} \mathrm{C}$. After extensive washing, secondary Ab conjugated to HRP was added at a 1:5000 dilution in 5\% nonfat milk in Tris buffer and blots subjected to ECL detection (PerkinElmer Life Sciences, Boston, MA).

\section{Quantitative real-time PCR}

RNA extraction and reverse-transcription reactions were carried out as previously described [27]. The cDNA primers used for PCR amplification were as follows: human TTP forward primer: TACACCATGGATCTGACTGC; reverse primer: TTACTCAGAAACAGAGATGC, human c-Jun forward primer: TGACTGCAAAGATGGAAACG; reverse primer: CAGGGTCATGCT CTGTTT CA, human Wee1 forward primer: GGCTCTGTTGATGAGCAGAACGCTT3, reverse primer: CTCAAGCCTCGGCGGCCAACTTGC. All measurements were performed in triplicate.

\section{Primary transcript measurement}

To determine primary transcript rates of c-Jun gene, cDNAs were synthesized with random primers with $1 \mu \mathrm{g}$ DNase-treated RNA. The primers used for the primary transcript were as follows: forward: CTGAGAGCGACGCGAGCCAAT and reverse: CTGGCTGTGTCTGTCTGTC. qRT-PCR was performed by a modified protocol as described previously [29].

\section{Adenoviral transduction}

Tumor-specific TTP expressing adenovirus 5 (Ad5) was generated by replacing the ADP gene with human TTP cDNA at the E3 immunoregulatory region in the backbone of the Ad5 vector having human telomerase reverse transcriptase promoter (hTERT) in the place of the E4 promoter. This TTP/Ad5 only replicates efficiently in highly dividing tumor cells but not normal cells [30]. Control Ad5 was generated by cleaving the
ADP in the same vector. Cells were infected for $4 \sim 6 \mathrm{~h}$ with different multiplicity of infection $(1: 1$ or $1: 5)$ in incomplete medium, followed by adding $20 \%$ serum into the medium. Cell proliferation assays and Western Blots were performed $24 \mathrm{~h}$ or $48 \mathrm{~h}$ after infection.

\section{Luciferase assay}

Transient transfections were performed as described previously [29]. Briefly, cell suspension was mixed with 5-8 $\mu \mathrm{g}$ total DNA (including reporter, effector, internal, control, and carrier DNA) and electroporated at 975 microfarad and $280 \mathrm{~V}$ in RPMI 1640 medium without serum. Luciferase activity was measured in cell lysates after 24 hours. All luciferase assays represent at least three independent experiments, each consisting of three wells per transfection. Transfected cells were used to extract protein for measurement of protein expression as well.

\section{Cell cycle analysis}

Cells were trypsinized, washed, and then resuspended in PBS. 100\% ethanol was added drop-wise to obtain a final ethanol concentration of $75 \%$. Cells were centrifuged at $2,000 \mathrm{rpm}$ at $4^{\circ} \mathrm{C}$ for 2 minutes, washed with PBS, and resuspended in PI working solution (PBS containing $1 \% \mathrm{FBS}, 250 \mu \mathrm{g} / \mathrm{ml}$ of RNase A, $30 \mu \mathrm{g} / \mathrm{ml}$ of PI). Cells were filtered through a $35 \mu \mathrm{m}$ strainer cap (Becton Dickinson, Franklin Lakes, NJ, USA) before being subjected to fluorescence-activated cell sorting (FACS) analysis.

\section{FACS}

$1 \times 10^{6}$ cells were resuspended in $1 \mathrm{ml}$ of binding buffer (PBS with $\mathrm{Ca} 2^{+} 0.33 \mathrm{~g} / \mathrm{L}$ to PBS), double stained with Annexin V/PI (1:10,000 dilution in PBS) and analyzed by flow cytometry (LSRII, BD Biosciences). Apoptotic cells were identified in the Annexin V positive/ PI negative quadrant. Data were processed with FACS Diva Software v6.1.2.

\section{Statistical analysis}

A Student's $t$ test was performed whenever applicable. SD of the mean is shown unless otherwise indicated. $p$ value $<0.05$ was considered statistically significant. *: $p<0.05$; **: $p<0.01$; ***: $p<0.001$ between two groups.

\section{ACKNOWLEDGMENTS}

The authors have no financial conflict of interest. We thank Perry Blackshear (National Institute of 
Environmental Health Sciences, Durham, NC) for providing us the expression vectors for CMV hTTP.Flag, CMVhTTP/C124R, CMVhTTP/C147R mutant; and K. Murphy (Washington University, St. Louis, MO) for the expression vectors for NF-кB p50, p65, and c-Rel. We also thank Karoly Toth (Saint Louis University) for assistance in generating the TTP/Adenovirus, and Daniel F. Hoft (Saint Louis University) for insightful discussion on the project.

\section{CONFLICTS OF INTEREST}

All authors have no conflict of interest in this study.

\section{GRANT SUPPORT}

This work was supported by the National Cancer Institute of the National Institutes of Health under Award Number R01CA163808 to Jianguo Liu and R01CA102428 to Gerald Wilson. The content is solely the responsibility of the authors and does not necessarily represent the official views of the National Institutes of Health.

\section{REFERENCES}

1. Hanahan D and Weinberg RA. Hallmarks of cancer: the next generation. Cell. 2011; 144(5):646-674.

2. Igney FH and Krammer PH. Death and anti-death: tumour resistance to apoptosis. Nat Rev Cancer. 2002; 2(4):277288.

3. Carballo E, Lai WS and Blackshear PJ. Feedback inhibition of macrophage tumor necrosis factor-alpha production by tristetraprolin. Science. 1998; 281(5379):1001-1005.

4. Sanduja S, Blanco FF, Young LE, Kaza V and Dixon DA. The role of tristetraprolin in cancer and inflammation. Front Biosci. 2012; 17:174-188.

5. Carballo E, Lai WS and Blackshear PJ. Evidence that tristetraprolin is a physiological regulator of granulocytemacrophage colony-stimulating factor messenger RNA deadenylation and stability. Blood. 2000; 95(6):1891-1899.

6. Qian X, Ning H, Zhang J, Hoft DF, Stumpo DJ, Blackshear PJ and Liu J. Posttranscriptional regulation of IL-23 expression by IFN-gamma through tristetraprolin. J Immunol. 2011; 186(11):6454-6464.

7. Phillips K, Kedersha N, Shen L, Blackshear PJ and Anderson P. Arthritis suppressor genes TIA-1 and TTP dampen the expression of tumor necrosis factor alpha, cyclooxygenase 2, and inflammatory arthritis. Proc Natl Acad Sci U S A. 2004; 101(7):2011-2016.

8. Taylor GA, Carballo E, Lee DM, Lai WS, Thompson MJ, Patel DD, Schenkman DI, Gilkeson GS, Broxmeyer HE, Haynes BF and Blackshear PJ. A pathogenetic role for TNF alpha in the syndrome of cachexia, arthritis, and autoimmunity resulting from tristetraprolin (TTP) deficiency. Immunity. 1996; 4(5):445-454.

9. Molle C, Zhang T, Ysebrant de Lendonck L, Gueydan C, Andrianne M, Sherer F, Van Simaeys G, Blackshear PJ, Leo $\mathrm{O}$ and Goriely S. Tristetraprolin regulation of interleukin 23 mRNA stability prevents a spontaneous inflammatory disease. J Exp Med. 2013; 210(9):1675-1684.

10. Carrick DM and Blackshear PJ. Comparative expression of tristetraprolin (TTP) family member transcripts in normal human tissues and cancer cell lines. Arch Biochem Biophys. 2007; 462(2):278-285.

11. Brennan SE, Kuwano Y, Alkharouf N, Blackshear PJ, Gorospe $\mathrm{M}$ and Wilson GM. The mRNA-destabilizing protein tristetraprolin is suppressed in many cancers, altering tumorigenic phenotypes and patient prognosis. Cancer Res. 2009; 69(12):5168-5176.

12. Suswam E, Li Y, Zhang X, Gillespie GY, Li X, Shacka JJ, Lu L, Zheng L and King PH. Tristetraprolin down-regulates interleukin-8 and vascular endothelial growth factor in malignant glioma cells. Cancer Res. 2008; 68(3):674-682.

13. Cha HJ, Lee HH, Chae SW, Cho WJ, Kim YM, Choi HJ, Choi DH, Jung SW, Min YJ, Lee BJ, Park SE and Park JW. Tristetraprolin downregulates the expression of both VEGF and COX-2 in human colon cancer. Hepatogastroenterology. 2011; 58(107-108):790-795.

14. Fahling $\mathrm{M}$, Persson AB, Klinger B, Benko E, Steege A, Kasim M, Patzak A, Persson PB, Wolf G, Bluthgen N and Mrowka R. Multilevel regulation of HIF-1 signaling by TTP. Mol Biol Cell. 2012; 23(20):4129-4141.

15. Al-Souhibani N, Al-Ahmadi W, Hesketh JE, Blackshear PJ and Khabar KS. The RNA-binding zinc-finger protein tristetraprolin regulates AU-rich mRNAs involved in breast cancer-related processes. Oncogene. 2010; 29(29):42054215.

16. Silverman $\mathrm{N}$ and Maniatis $\mathrm{T}$. NF-kappaB signaling pathways in mammalian and insect innate immunity. Genes Dev. 2001; 15(18):2321-2342.

17. Perkins ND. Integrating cell-signalling pathways with NFkappaB and IKK function. Nat Rev Mol Cell Biol. 2007; $8(1): 49-62$.

18. Gilmore TD. Introduction to NF-kappaB: players, pathways, perspectives. Oncogene. 2006; 25(51):6680-6684.

19. Shaulian E and Karin M. AP-1 as a regulator of cell life and death. Nat Cell Biol. 2002; 4(5):E131-136.

20. Lopez-Bergami P, Lau E and Ronai Z. Emerging roles of ATF2 and the dynamic AP1 network in cancer. Nat Rev Cancer. 2010; 10(1):65-76.

21. Tiniakos DG, Scott LE, Corbett IP, Piggott NH and Horne $\mathrm{CH}$. Studies of c-jun oncogene expression in human breast using a new monoclonal antibody, NCL-DK4. J Pathol. 1994; 172(1):19-26.

22. Yokoyama Y, Sagara M, Sato S and Saito Y. Value of glutathione S-transferase pi and the oncogene products c-Jun, c-Fos, c-H-Ras, and c-Myc as a prognostic indicator in endometrial carcinomas. Gynecol Oncol. 1998; 
68(3):280-287.

23. Yang L, Kim HT, Munoz-Medellin D, Reddy P and Brown $\mathrm{PH}$. Induction of retinoid resistance in breast cancer cells by overexpression of cJun. Cancer Res. 1997; 57(20):46524661.

24. Smith LM, Wise SC, Hendricks DT, Sabichi AL, Bos T, Reddy P, Brown PH and Birrer MJ. cJun overexpression in MCF-7 breast cancer cells produces a tumorigenic, invasive and hormone resistant phenotype. Oncogene. 1999; 18(44):6063-6070.

25. Jiao X, Katiyar S, Willmarth NE, Liu M, Ma X, Flomenberg $\mathrm{N}$, Lisanti MP and Pestell RG. c-Jun induces mammary epithelial cellular invasion and breast cancer stem cell expansion. J Biol Chem. 2010; 285(11):8218-8226.

26. Lai WS, Carballo E, Strum JR, Kennington EA, Phillips RS and Blackshear PJ. Evidence that tristetraprolin binds to AU-rich elements and promotes the deadenylation and destabilization of tumor necrosis factor alpha mRNA. Mol Cell Biol. 1999; 19(6):4311-4323.

27. Murphy TL, Cleveland MG, Kulesza P, Magram J and Murphy KM. Regulation of interleukin 12 p40 expression through an NF-kappa B half-site. Mol Cell Biol. 1995; 15(10):5258-5267.

28. Liu J, Cao S, Herman LM and Ma X. Differential regulation of interleukin (IL)-12 p35 and p40 gene expression and interferon (IFN)-gamma-primed IL-12 production by IFN regulatory factor 1. J Exp Med. 2003; 198(8):1265-1276.

29. Zhang J, Qian X, Ning H, Yang J, Xiong H and Liu J. Activation of IL-27 p28 gene transcription by interferon regulatory factor 8 in cooperation with interferon regulatory factor 1. J Biol Chem. 2010; 285(28):21269-21281.

30. Kuppuswamy M, Spencer JF, Doronin K, Tollefson AE, Wold WS and Toth K. Oncolytic adenovirus that overproduces ADP and replicates selectively in tumors due to hTERT promoter-regulated E4 gene expression. Gene Ther. 2005; 12(22):1608-1617.

31. Wisdom R, Johnson RS and Moore C. c-Jun regulates cell cycle progression and apoptosis by distinct mechanisms. EMBO J. 1999; 18(1):188-197.

32. Schreiber M, Kolbus A, Piu F, Szabowski A, MohleSteinlein U, Tian J, Karin M, Angel $P$ and Wagner EF. Control of cell cycle progression by c-Jun is p53 dependent. Genes Dev. 1999; 13(5):607-619.

33. Hudson BP, Martinez-Yamout MA, Dyson HJ and Wright PE. Recognition of the mRNA AU-rich element by the zinc finger domain of TIS11d. Nat Struct Mol Biol. 2004; 11(3):257-264.

34. Lai WS, Kennington EA and Blackshear PJ. Interactions of $\mathrm{CCCH}$ zinc finger proteins with mRNA: non-binding tristetraprolin mutants exert an inhibitory effect on degradation of AU-rich element-containing mRNAs. J Biol Chem. 2002; 277(11):9606-9613.

35. Hake LE, Mendez R and Richter JD. Specificity of RNA binding by CPEB: requirement for RNA recognition motifs and a novel zinc finger. Mol Cell Biol. 1998; 18(2):685693.

36. Schichl YM, Resch U, Lemberger CE, Stichlberger D and de Martin R. Novel phosphorylation-dependent ubiquitination of tristetraprolin by mitogen-activated protein kinase/extracellular signal-regulated kinase kinase kinase 1 (MEKK1) and tumor necrosis factor receptor-associated factor 2 (TRAF2). J Biol Chem. 2011; 286(44):3846638477.

37. Chen YL, Jiang YW, Su YL, Lee SC, Chang MS and Chang CJ. Transcriptional regulation of tristetraprolin by NF-kappaB signaling in LPS-stimulated macrophages. Mol Biol Rep. 2013; 40(4):2867-2877.

38. Gu L, Ning H, Qian X, Huang Q, Hou R, Almourani R, Fu M, Blackshear PJ and Liu J. Suppression of IL-12 production by tristetraprolin through blocking NF-kcyB nuclear translocation. J Immunol. 2013; 191(7):3922-3930.

39. Griseri P, Bourcier C, Hieblot C, Essafi-Benkhadir K, Chamorey E, Touriol C and Pages G. A synonymous polymorphism of the Tristetraprolin (TTP) gene, an AUrich mRNA-binding protein, affects translation efficiency and response to Herceptin treatment in breast cancer patients. Hum Mol Genet. 2011; 20(23):4556-4568.

40. Ishmael FT, Fang X, Galdiero MR, Atasoy U, Rigby WF, Gorospe M, Cheadle C and Stellato C. Role of the RNAbinding protein tristetraprolin in glucocorticoid-mediated gene regulation. J Immunol. 2008; 180(12):8342-8353.

41. Schichl YM, Resch U, Hofer-Warbinek R and de Martin R. Tristetraprolin impairs NF-kappaB/p65 nuclear translocation. J Biol Chem. 2009; 284(43):29571-29581.

42. Barrios-Garcia T, Tecalco-Cruz A, Gomez-Romero V, Reyes-Carmona S, Meneses-Morales I and Leon-DelRio A. Tristetraprolin represses estrogen receptor alpha transactivation in breast cancer cells. J Biol Chem. 2014; 289(22):15554-15565.

43. Al-Haj L, Blackshear PJ and Khabar KS. Regulation of p21/CIP1/WAF-1 mediated cell-cycle arrest by RNase L and tristetraprolin, and involvement of AU-rich elements. Nucleic Acids Res. 2012; 40(16):7739-7752.

44. Marderosian M, Sharma A, Funk AP, Vartanian R, Masri J, Jo OD and Gera JF. Tristetraprolin regulates Cyclin D1 and c-Myc mRNA stability in response to rapamycin in an Aktdependent manner via p38 MAPK signaling. Oncogene. 2006; 25(47):6277-6290.

45. Lee HH, Lee SR and Leem SH. Tristetraprolin regulates prostate cancer cell growth through suppression of E2F1. J Microbiol Biotechnol. 2014; 24(2):287-294.

46. Lee HH, Vo MT, Kim HJ, Lee UH, Kim CW, Kim HK, Ko MS, Lee WH, Cha SJ, Min YJ, Choi DH, Suh HS, Lee BJ, Park JW and Cho WJ. Stability of the LATS2 tumor suppressor gene is regulated by tristetraprolin. J Biol Chem. 2010; 285(23):17329-17337.

47. Russell $P$ and Nurse $P$. Negative regulation of mitosis by wee1+, a gene encoding a protein kinase homolog. Cell. 
1987; 49(4):559-567.

48. Parker LL and Piwnica-Worms H. Inactivation of the p34cdc2-cyclin B complex by the human WEE1 tyrosine kinase. Science. 1992; 257(5078):1955-1957.

49. Liang J, Lei T, Song Y, Yanes N, Qi Y and Fu M. RNAdestabilizing factor tristetraprolin negatively regulates NF-kappaB signaling. J Biol Chem. 2009; 284(43):2938329390.

50. Lal S, Burkhart RA, Beeharry N, Bhattacharjee V, Londin ER, Cozzitorto JA, Romeo C, Jimbo M, Norris ZA, Yeo CJ, Sawicki JA, Winter JM, Rigoutsos I, Yen TJ and Brody JR. HuR posttranscriptionally regulates WEE1: implications for the DNA damage response in pancreatic cancer cells. Cancer Res. 2014; 74(4):1128-1140. 